\title{
Semantic Integration for Cross-organizational Manufacturing Business Process
}

\author{
Wang Mingwei*, Li Shan, Zhou Jingtao and Zhang Shusheng
}

The Key Laboratory of Contemporary Design and Integrated Manufacturing Technology Ministry of Education,
Northwestern Polytechnical University, Xi'an, Shaan'xi, China

\begin{abstract}
Enhanced the business-to-business process integration capabilities is a key success factor in manufacturing industry. However, challenges such as changing markets and heterogeneous manufacturing resources counteract business process interactions. This paper proposes an integration framework for cross-organizational processes, in which two technologies, namely ontology and web service composition, are applied throughout process modeling, implementation and execution. Various manufacturing resources are encapsulated into uniform manufacturing services whose capabilities including properties and interfaces are encoded in an unambiguous as well as computer-understandable form. Leveraging the capabilities profiles as the mediation in the framework, business logic and implementation techniques are loosely coupling relationship, which enable enterprises to collaborate without prior relationship established and to adjust their processes on demand. Three key implementation techniques: semantic modeling of manufacturing services, services discovery and optimization selection are discussed in detailed.
\end{abstract}

Keywords: Business process integration, manufacturing service, semantic web, web service composition.

\section{INTRODUCTION}

In the context of global markets, manufacturing industry has two features: highly specialized division of labor and highly degree of collaboration. Each enterprise only focuses on his core competencies and products (or parts), while the tasks of non-core sub-components are assigned to their distributed partners. In order to organize these scattered resources together, the enterprise need to create external collaborative business processes with multiple partners during which all kinds of competencies and resources are optimized the allocation. Furthermore, the enterprise must try hard to adjust their production processes in a timely to respond to changes in markets.

Traditional process integration methods like Enterprise Application Integration (EAI) [1] focus on standard interfaces and formats for the information to flow from one application to another rather than on the actual information contents and logic, which have failed to address the requirement for spanning business lines and adapting quickly business processes changes under highly dynamic networked environment. The business semantic and principles of collaboration enterprises are scattered and hidden in a variety of customization interface codes, which causes a great deal of manual processing and recoding on established applications even if a trivial changes in business model such as adding a step [2].

With advances in service-oriented architecture (SOA) and related technologies, they offer the opportunity to

*Address correspondence to this author at the The Key Laboratory of Contemporary Design and Integrated Manufacturing Technology Ministry of Education, Northwestern Polytechnical University, Xi'an, Shaan'xi, China; Tel: 86-29-88493232-325; Fax: 86-29-88491576; E-mails: wangmv@nwpu.edu.cn,wang-tangtang@163.com develop the loosely coupled business integration framework. Base on discovery and selection of web service, executable business process can be created flexibly by composing existing services which are abstracted either from existed applications and data or from external manufacturing sources. However, current commercial web service architectures such as UDDI lacks an adequate and machine-readable specification of what the objects of the service, leading to inconsistent interpretations and interoperability.

Ontology has been developed to annotate capabilities and interfaces of services in unambiguous, computer-understandable forms, which provides rigorous definition for shared and common understanding of manufacturing domain. It will be possible to access service resources by content rather than just by keywords, and to discovery and composition service autonomously.

According to the special characteristics and requirements of cross-organizational manufacturing business processes, the objective of the paper is to describe a dynamic and adaptive integration framework based on ontology. The remainder of the paper is organized as follows. In section 2, the related works are investigated based on a short survey of current approaches. The functional requirements are discussing in section 3. A framework for manufacturing business process integration is discussed in section 4 . In section 5 and section 6, the basic principles and implication technologies of the solution are presented. Section 7 gives the concluding remarks.

\section{RELATED WORKS}

By means of embedding legacy applications into web services [3], the enterprise enable to integrate and manipulate data from distributed manufacturing sources without worrying about the underlying syntactical details. When compos- 
ing web services, the business logic of the enterprise is implemented. The method of web service composition facilitates resource sharing and business interoperability under networked manufacturing which has been the focus of much research recently, increased in business process integration within and across organizations. In this section, we will discuss the related work that deals with web service composition and other research efforts that are related to our work.

Symphnyo [4] and eFlow [5] have proposed business process integration approaches based on workflow-based composition of Web services, which firstly create a business workflow model and attach relative services to corresponding business activity based on service discovery. However, they are lacking in an important aspect. The semantics of business logic and web service is hidden in syntax description like WSDL files. There are much semantic incompatible problems on business processes, messages, and documents during process transactions. It leads services to be difficult to fully understood and reused in different scenarios. Also the activity of creating processes has been handled mostly at the syntactic level, which turns out low degree of process automation and dynamic adjustment $[6,7]$.

On the other hand, METEOR-S [8] and SHOP2 [9] aim to automate the entire composition process by using AI planning or semantic web technology. Ontology is used to annotate parameters, preconditions and results of web service, and the model of business process. So the business process can be automatically achieved by formal reasoning of these semantic models in theory. The method is highly automotive and intelligent, but it is difficult to use for clients because of the high complexity and the immature of composition of services relying solely on the computer.

Consequently, this paper fuses the two methods discussed above by using the semantic clues, proposing a semantic composition framework for business process integration to support intelligent distributed manufacturing, in which manufacturing resources are wrapped as semantic web services for remote invocation and loose-coupling coordination. The framework uses workflow-based service composition method as the base, which enable web service combined together according to business process logic. Moreover, our approach utilizes domain ontology to describe capacity of manufacturing resources and service discovery.

\section{FUNCTIONAL REQUIREMENTS}

In this section, we will outline general requirements for the integration framework. Typically, the melting of service oriented thinking and manufacturing characters greatly enhances the complexity of manufacturing systems. In addition to the same characteristics with normal manufacturing process such as complicated business logic, a wide variety of business, and large volume of data, compared with the traditional business process deployed in enterprise, collaborative cross-organizational business process must adhere to certain higher requirements.

1. Collaborative cross-organizational business process may span across several different enterprise and organizational. So the framework must be open and scalable which should make full use of known standard specifications and communication protocol.

2. In terms of production facilities, the enterprise can adjust the production process in a timely and optimal manner to respond rapidly to changes in demand or capacity. There must be mechanisms to achieve loosely coupled between the specific IT implementations and corresponding business logic.

3. Each process consists of many activities, which invoke complex manufacturing resources belonging to different organizations. These resources may be heterogeneous in syntax and semantic. So the framework should support resource sharing mechanism to resolve the heterogeneity.

4. The potential number of suitable services in dynamic and complex service-rich environments like the networked manufacturing can be extremely large. It would be unrealistic to expect a user to be able to locate a suitable service. The framework should provide a more efficient and automation mechanism of service discovery and composition to reduce the manual workload.

\section{THE FRAMEWORK}

In this paper, we propose an integration framework, called $\mathrm{S}^{2} \mathrm{BPC}$, based on workflow-based service composition and ontology. As shown in Fig. (1), $\mathrm{S}^{2} \mathrm{BPC}$ is composed of five layers: Business Model Layer (ML), Business Process Layer (PL), Manufacturing Services Layer (MSL), Manufacturing Resource Layer (RL) and Ontology Layer (OL). The semantic description of manufacturing services, namely service capability layer, puts together available services and user-specified preferences.

\section{1) Manufacturing Resource Layer}

This layer represents a collection of all shared resources of cooperative enterprises, including not only the physical factors such as equipment, tools and software, but also information and technical factors generated by the serving process such as design capacity information.

\section{2) Manufacturing Service Layer}

This layer is responsible for encapsulating all types of resources in ML into Manufacturing Service, which converts local resources into global sharing resources. Partners are supported to invoke others' resources across the Internet programmatically using standard Internet protocols and representation formats like Hypertext Transfer Protocol (HTTP) and Extensible Markup Language (XML). In addition, this layer provides the environment for manufacturing service modeling, publishing and operating. The heterogeneity problem of manufacturing resources is resolved in this layer by the semantic description of services based on the ontologies defined in OL.

In this paper, manufacturing service [10] is self-describing, self-contained, platform-independent software entity, which is generated from service virtualization and semantic annotation method for manufacturing resource, 


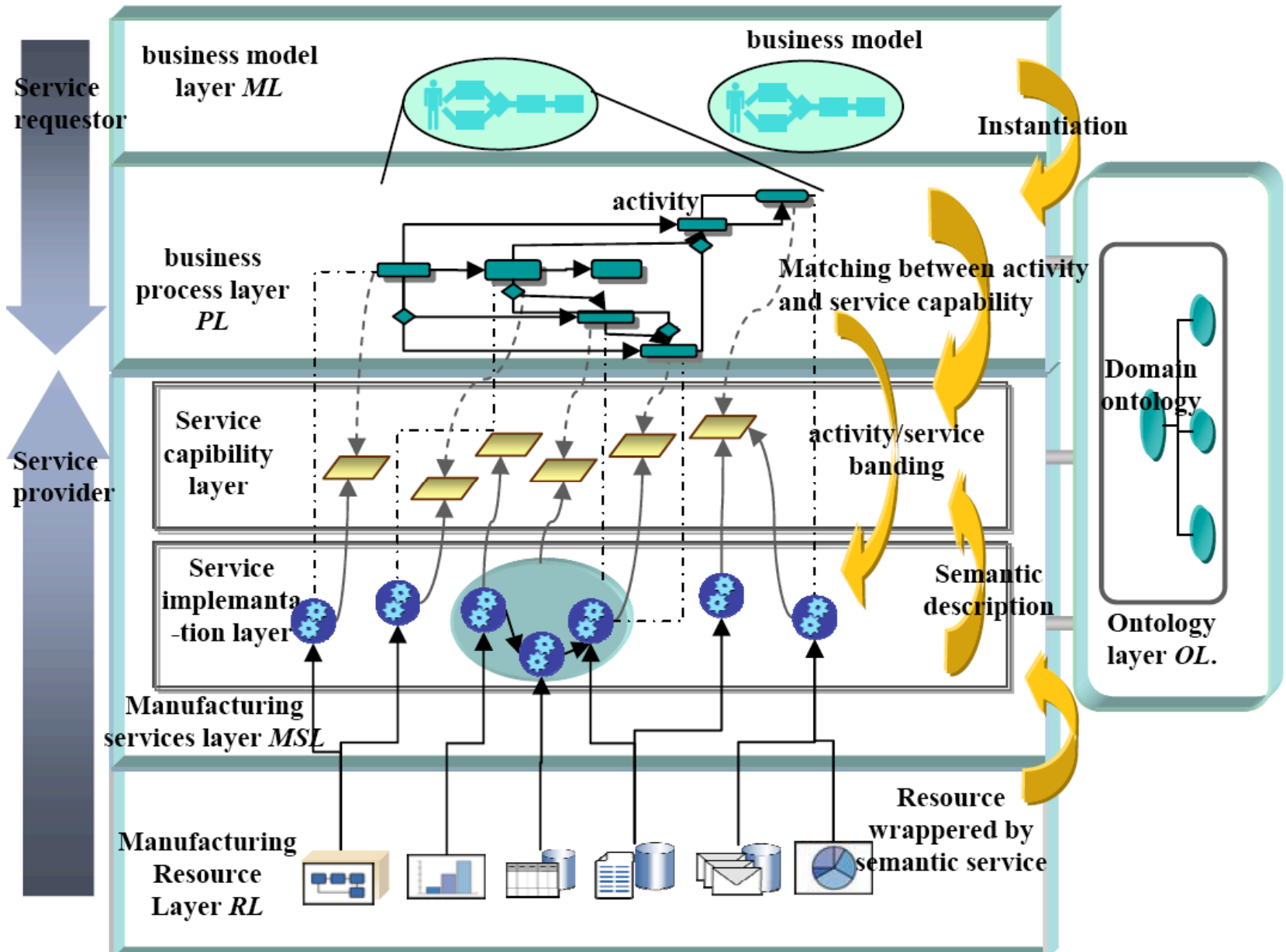

Fig. (1). $\mathrm{S}^{2} \mathrm{BPC}$ integration framework.

performs certain manufacturing functions and has a public access mechanism.

\section{Definition 1}

A Manufacturing Service $m s=\left(S O, M_{s o}, A\right)$ is exactly the same as semantic web service, where $S O$ is the service object entity including interfaces and realization codes using WSDL (Web Service Description Language) to achieve the functionality provided by wrapped manufacturing resources; $M_{s o}$ is the mapping between $S O$ and manufacturing resources (or $S O$ in other $m s$ ) in order to achieve encapsulation, distribution and invocation of the operation on manufacturing resources (or other $m s$ ); $A$ is the semantic description of $m s$ capability containing machining-readable services features, such as quality properties, whose information is restricted by semantic annotation using domain ontology defined in OL.

The collection of $S O$ of entire manufacturing services constitutes the service implementation layer (SOL), so do all $A$ constitutes the service capability layer (AL) at semantic level. Especially the "semantic level" means that two $m s$ is the same depending on whether their ability description $A$ is semantically identical.

\section{3) Business Model Layer}

This layer is responsible for describing the collaborative relationships of business activities in business transactions. It is the basis for the operation and control of collaborative business processes.

\section{Definition 2}

A business model MPM= $\left(M A, F_{M A-M A}\right)$ is a resourceindependent process plan, where $M A$ is a finite, non-empty set whose member, namely manufacturing activity ( $m a$ ), specifies requirements for the manufacturing goal, such as quality requirements, input / output parameters; $F_{M A-M A} \subseteq$ $M A \times M A-I_{\mathrm{MA}}$ is a non-cycle relation, used to represent the business synergies between $m a$, and $I_{M A}$ is the identical relation.

\section{4) Business Process Layer}

This layer is responsible for the establishment of mappings between manufacturing activities and manufacturing services. The partial order sequence of services will be set up and executed. In fact, one business process is an instance of MPM that each $m a$ is attached with a single $m s$. Manufacturers and suppliers are transparent of the underlying processes and computing environments of the participants by discovering, orchestrating, and invocating of $m s$. This mapping is realized by the semantic discovering and matching between manufacturing activity and service semantic description. 


\section{5) Ontology Layer}

The layer is made up of a number of ontologies for manufacturing domain, such as TOVE (TOronto Virtual Enterprise). Each ontology provides a shared terminology for the particular domain that each enterprises can jointly understand and use. This layer provides semantic reference basis for computational representation of manufacturing activities and capabilities of manufacturing services, which minimize ambiguity and maximize understanding and precision in communication in different business scenes.

\section{BASIC PRINCIPLE}

With the support of our framework, the service provider can share their resources and knowledge by means of encapsulating them into manufacturing services, and the partner with demand for manufacturing task (as a service requester) can integrate dynamically their collaborative process by business process modeling, service discovery and service composition. Functional interaction and data access between enterprises have been converted to request and invocation of manufacturing services. Our approach shields the heterogeneous and complexity within the manufacturing resources, and improves the agility and maintainability of the construction procedure of collaborative business process.

The framework is characterized as loosely integrated between $m a$ and $m s$. From the definition of business model layer, our framework has not designated any fixed service providers for manufacturing activity in the course of business modeling. The dynamic association between business activity and specific service is achieved by using service capabilities as an intermediary to invoke the service implementation $S O$.

In order to achieve the goal, we have conducted semantic match and discovery between service request description, namely manufacturing activity definition $m a$, and service capabilities advertising $m s$. A. Matching result is a manufacturing services set $U_{H}$ in which each service has equal capability to satisfy $m a$. After resource service optimal selection and composition, the ability reference relationship $F_{M A-A L}$ between $m a$ and $m s\left(m s \in U_{H}\right)$ could be set up.

During the business modeling process, the service requestor only need to consider how to define the accurate sequential logic constrains among manufacturing activities from a global view of product development without considering whether the activities across enterprises' borders or by which manufacturing resources to complete the task. If the business logic is adjusted, it only needs to change the $F_{\text {MA-AL, and }}$ will not affect the implementation of manufacturing service (i.e., $S O$ ). On the other hand, changes in the ways by which the manufacturing service is implemented will not affect the definition of business models and other services.

Therefore, the abstract service capabilities layer is acting as the coordination center between manufacturing activity and service implementation (ref. Fig. 1), which reduces the coupling degree of business logic and specific implementation, enhancing the dynamic adjustment capabilities of collaborative business process. Meanwhile, matching / selection/binding process of manufacturing services can make use of automatic processing of semantic reasoning techniques to improve the automation and intelligence of the procedure for building collaborative business process.

\section{KEY IMPLEMENTATION TECHNIQUES}

As introduced above of the basic principle of collaborative business process integration, four primary issues are implemented including business process modeling, manufacturing services modeling, manufacturing services discovery and manufacturing services composition. The business process modeling has been researched much in workflow area. We adopts extended activity network diagram method [11] as business process modeling method for our framework that will no longer be mentioned here. In this section, we mainly discuss remaining three implementation techniques.

\subsection{Semantic Model of Manufacturing Service}

At the start of business collaboration, an enterprise needs to find other partners that may be able to provide a required service. Manufacturing capability profile, namely semantic description of manufacturing service capability $A$ in definition 1, allows the service provider to advertise their sharing resources and knowledge. Manufacturing capability profiles are stored in open registries like UDDI that provides human and computer interfaces to register services and to search for them.

\section{Definition 3}

A manufacturing service profile $A=($ Property, ECapacity, Interface, Quality) is a four tuples, where Property is the set of general service information such as name, provider and location (e.g., URL); ECapacity is the set of what kind of problems can be solved; Interface is the set of input and output parameters; and Quality is the set of non-functional properties such as cost and time.

Obviously, ECapacity is the core of the profile, which describes special engineering and business information that normal web service or semantic web service has yet not addressed. From the external point of view, i.e., that of a service requester who don't care about the comprehensive description of the internal structure of manufacturing resources, the manufacturing service is seen as a black box that exhibit a certain exported behavior on some products. When executed, the manufacturing service can perform its task by directly invoking encapsulated manufacturing resources and using certain methods under certain conditions. Theses five factors are more concerned information in the collaborative manufacturing than others. So, ECapacity is defined as Ecapacity $=$ (product, method, resource, condition, task).

Each set in $A$ is finite and non-empty in which each element is realized based on extended OWL-S [12] and manufacturing domain ontologies. As shown in Fig. (2) [10], the sets of Property and Interface are still represented by ServiceModel and ServiceGrouding. We extend OWL-S with two subclasses: ManufacturingProfile and QoSProfile to specify ECapacity and Quality.

The ManufacturingProfile class defines five properties: hasProduct, hasResource, hasMethod, hasTask and hasConditon to describe the manufacturing capability, 

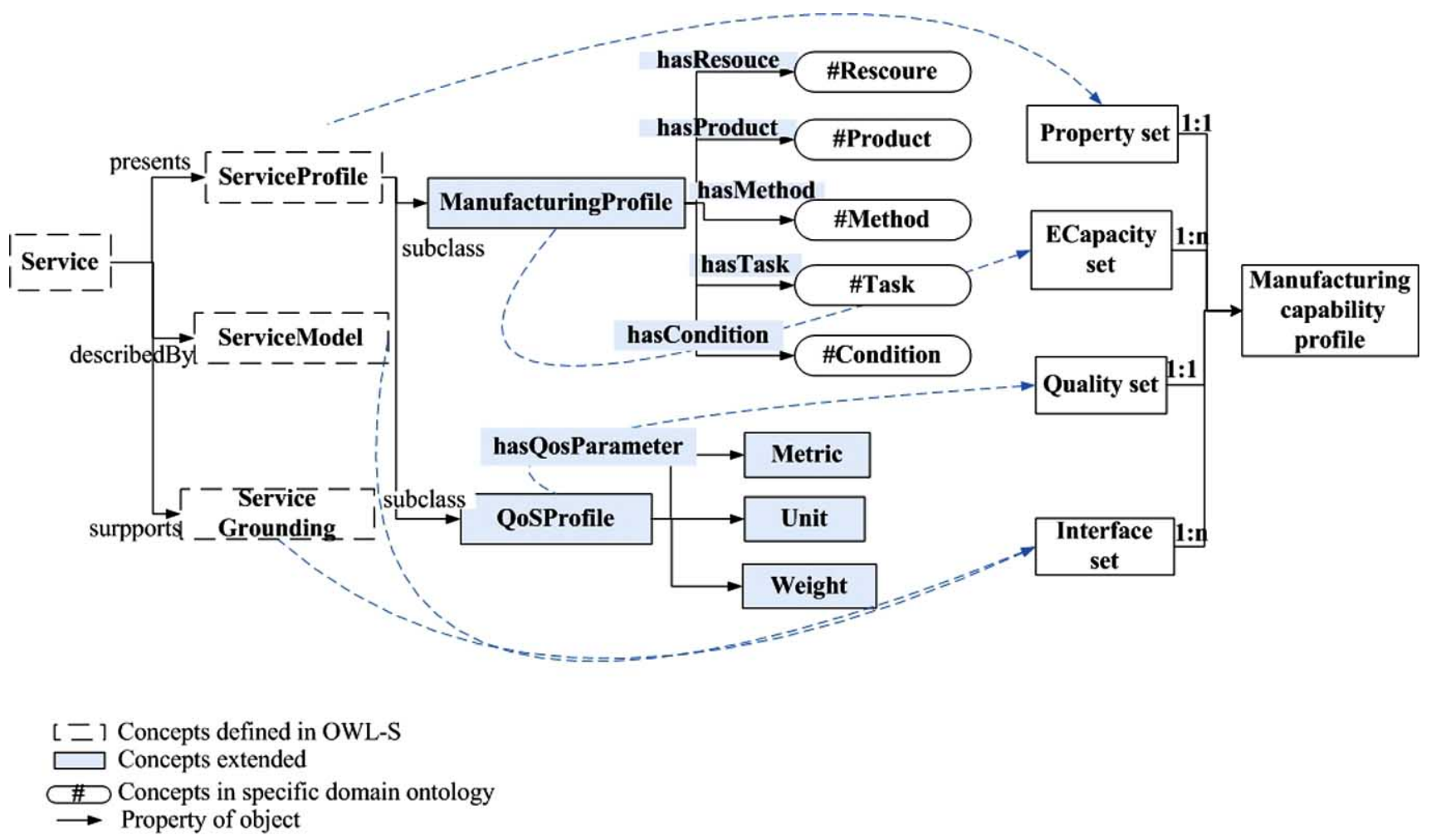

Fig. (2). The extension of OWL-S.

namely Ecapacity. The five properties range over instances as defined in the manufacturing domain ontologies like TOVE, Enterprise Ontology and Core Enterprise Ontology.

The QoSProfile class with three basic classes to provide semantic markups for explicitly describing QoS(quality of service) information. The Metric class defines QoS measuring parameters. The Unit class defines the measurement units of Metric. The Weight class defines the ponderance that the requester concerns with some Metric. The QoSProfile class is parent class for all the QoS properties. One service can be described by at most one QoSProfile instance.

\subsection{Service Discovery Based on Semantically Matching}

In order to realize effectively manufacturing service match and search, a variety of papers dealing with service discovery have recently been published. The key object of these algorithms is to evaluate similarity degree between service requests and advertisings. Based on shared ontologies to provide semantic understanding, our algorithm [13] has adopted to calculate the semantic similarity degree with digital value, not key words similarity, to measure correlation between the service request $m a$ and manufacturing service profile $m s . A$. Thus, service discovery is a procedure to find a subset $U_{H}$ from $U_{A}$ that is set of all services profiles. The semantic similarity degree in $U_{\mathrm{H}}$ is greater than or equal to threshold value $\omega_{r}$ service requester specified, as formulated in expression 1 where $\operatorname{Match}(\mathrm{ma} . \mathrm{A}, \mathrm{ms})$ is the function to measure the match degree between $m a$ and $m s . A$.

$U_{H}=\left\{s \mid s \in U_{A} \wedge \operatorname{Match}(\operatorname{ma} . A, m s) \geq \omega_{r}\right\}$
Due to the definition of manufacturing service profile in 6.1, a three-phase service match and search method is proposed including ECapacit matching, I/O matching and QoS matching. The Match $(m a . A, m s)$ is defined as follows:

$$
\begin{array}{r}
\operatorname{Match}(m a, m s)=\prod\left(\operatorname{Match}_{e c}(m a, m s . A), \operatorname{Match}_{i / o}(m a, m s . A),\right. \\
\left.\operatorname{Match}_{q o s}(m a, m s . A)\right)
\end{array}
$$

Where:

- Match $_{e c}(m a . A, m s)$ denotes ECapacity matching. It is primarily responsible for matching the manufacturing capacities information between $m a$ and $m s$, such as hasproduct, which requires high precision matching. The detailed methods and algorithms are shown in [14]. The matchmaking result is divided into five types: exact match, plug-in match, subsume match, intersection match, and mismatch. In this phase we set the basic threshold value $\omega_{r}$ is "subsume match". If the result of ECapacity matching is lower than $\omega_{r}$, the algorithm will stop further matching and returns the failed message to use, which can improve discovery speed.

- $\operatorname{Match}_{i / o}(m a . A, m s)$ is the $\mathrm{I} / \mathrm{O}$ matching, i.e. inputs and outputs matching. It is primarily responsible for matching parameters of inputs and outputs. Because different data types of parameters may be converted each other, Match $_{i / o}(m a . A, m s)$ does not require high precision, but high recall. In this phase similarity degree is calculated according to Tversky's model [15] and Andrea' similarity functions [16]. The threshold value $\omega_{r}$ points interval $[0,1]$ that is set by the service requester. 
- $\operatorname{Match}_{\text {qos }}(m a . A, m s)$ is QoS matching. It is primarily responsible for matching QoS parameters such as time, cost and trust. The threshold value $\omega_{r}$ could be specified via the metricvalue property shown in section 6.1. The detailed methods and algorithms are shown in [17].

If each match result is allowed numerical interval, this means the manufacturing service is qualified for all the requirements and can be selected form $U_{A}$ for executing the requested task. The ECapacity matching, I/O matching, QoS matching can be used separately or together, which is decided by the practical requirements and conditions of user or system.

\subsection{Business Processes Generating Based on Service Optimization Selection}

After service matching stage, there may be many manufacturing services in $U_{H}$ which all meet the functional requirements of manufacturing activities. But they have different QoS parameters. Therefore, the system needs to choose a specific service to link other selected services together to form an executable services composition process. To achieve multi-QoS constraints, service selection with QoS global optimization is a NP-complete problem. We has adopted algorithms are shown [18]. The essence of the algorithm is to transform the problem into a multi-objective services composition optimization with QoS constraints. The algorithm took cost and time for example to be two optimal objective criteria, which is formulated as:

$\operatorname{MinF}(P)=(T(P), C(P))$

The path $P$ in the formula 3 subjects to two constraints: reputation and reliability, which is formulated as:

$$
\left\{\begin{array}{l}
\operatorname{Rep}(P) \geq \operatorname{Rep}_{0} \\
R(P) \geq R_{0}
\end{array}\right.
$$

$T(P), C(P), \operatorname{Rep}(P)$ and $R(P)$ are QoS parameters calculating functions. $\operatorname{Rep}_{0}$ and $R_{0}$ represent the required minimum reputation and the lowest reliability of composition solution.

The theory of intelligent optimization of multi-objective genetic algorithm was utilized to produce a set of optimal pareto services composition process with constraint principle by means of optimizing various objective functions simultaneously. Thus the service requester can choose the most satisfactory solution based on specific needs. The other composition solutions which are not selected are taken as alternatives and will be enabled when the selected path is in the event of accident. Theoretical analysis and experimental results indicated the feasibility and efficiency of this algorithm.

\section{CONCLUSIONS}

In this paper, an integration framework based on ontology and web services composition was proposed to implement collaborative cross-organizational business process in networked manufacturing environment. We leveraged SOA concepts by encapsulating various manufacturing resources into uniform manufacturing services, which were function operations for business activities. The service capacity was semantically annotated by extended OWL-S. With service capacity profile taking as the mediation, loose coupling relationship between business activities and implementation techniques was achieved. Partners can collaborate without prior relationship established. Three primary implement techniques were introduced as well. Although we have given a functional view of the framework and its mechanism, there are many key areas for our future research, such as manufacturing domain ontology definition, process on-line monitoring and dispatching.

\section{ACKNOWLEDGEMENTS}

The work is supported by National 863/CIMS of China through approval no. 2009AA04Z122, by National Natural Science Foundation of China through approval no. 70931004 and by China Postdoctoral Science Foundation through approval no. 201003681.

\section{REFERENCES}

[1] D. S. Linthicum, Enterprise Application Integration, AddisonWesley Longman Ltd, 2000.

[2] J. Bin, Y. Guangrong, and Z. Xinxiong, "SOA-based business process integration system for small and medium-sized manufacturing enterprises", Journal of Computer-Aided Design \& Computer Graphics, vol. 19, pp. 125-129, 2007.

[3] K. Czajkowski, D. F. Ferguson, I. Foster, J. Frey, S. Graham, I. Sedukhin, D. Snelling, S. Tuecke, and W. Vambenepe, "The WSresource framework Version 1.0", [Online], Available from: http://www.oasis-open.org/committees/download.php/16654/wsrfcs-01.zip [Accessed March 29 2010].

[4] G. Chafle, S. Chandra, and V. Mnna, "Decentralized orchestration of composite web services", In: 13th International World Wide Web Conference, New York, 2004, pp. 134-143.

[5] F. Casati, and M. C. Shan, "Event-based interaction management for composite e-services in eflow", Information Systems Frontiers, vol. 4, pp. 19-32, 2002.

[6] Z. Ma, B. Wetzstein, and D. Ani, "Semantic business process management: a lifecycle based requirements analysis", In: Proc of Workshops on Semantic Business Process and Product Lifecycle Management, 2007, pp. 1-11.

[7] G. Xuejun, Q. Xuejie, and D. Chun, "Research and implementation of business process integration based on semantic web services", Computer Technology and Development, vol. 16, pp. 106-109, 2006

[8] K. Verma, K. Gomadam, A. P. Sheth, J. A. Miller, and Z. Wu, "The METEOR-S approach for configuring and executing dynamic web processes", Technical Report, [Online], Available from: http://sdis.cs.uga.edu/projects/meteor-s/techRep6-24-05.pdf, [Accessed Sep 16 2007].

[9] U. Kuter, E. Sirin, B. Parsia, D. Nau, and J. Hendler, "Information gathering during planning for web service composition", In: Proc of Web Semantic: Science, Services and Agents on the World Wide Web, 2005, pp. 183-205.

[10] W. Mingwei, Z. Shusheng, and Z. Jingtao, "Semantic web service based framework for inter-organizational business process management", Advanced Materials Research, vol. 156-157, pp. 915-918, 2011

[11] H. Yuanyuan, and G. Chunming, "Research of workflow process definition tool based on activity-network-based workflow diagram", Computer Engineering and Applications, vol. 2, pp. 48-51, 2006.

[12] OWL-S: Semantic Markup for Web Services, [Online], Available: http://www.w3.org/Submission/2004/SUBM-OWL-S-20041122/

[13] W. Mingwei, Z. Shusheng, Z. Jingtao, and Z. Han, "An efficient method of semantic discovery of enterprise information integration using hybrid schema matching strategy", Journal of Northwestern Polytechnical University, vol. 27, pp. 590-594, 2009.

[14] D. Bianchini, V. DeAntonellis, and M. Melchiori, "An ontology based architecture for service discovery and advice system", In: 
Proc of the 16th International Workshop on Database and Expert Systems Applications, 2005, pp. 551-556.

[15] A. Tversky, "Features of similarity", Psychology Reviews, vol. 84, pp. 327-352, 1977.

[16] M. A. Rodriguez, and M. J. Egenhofer, "Determining semantic similarity among entity classes from different ontologies", IEEE Transactions on Knowledge and Data Engineering, vol. 15, pp. 442-456, 2003.
[17] F. Tao, Y. Hu, and D. Zhao, "Study on resource service match and search in manufacturing grid system", The International Journal of Advanced Manufacturing Technology, vol. 43, pp. 379-399, 2009.

[18] L. ShuLei, L. YunXiang, Z. Fan, T. GuiFen, and J. Ning, "A dynamic web services selection algorithm with QoS global optimal in web services composition", Journal of Software, vol. 18, pp. 646$656,2007$.

(C) Mingwei et al.; Licensee Bentham Open.

This is an open access article licensed under the terms of the Creative Commons Attribution Non-Commercial License (http: //creativecommons.org/licenses/bync/3.0/), which permits unrestricted, non-commercial use, distribution and reproduction in any medium, provided the work is properly cited. 